\title{
Acute cortical blindness caused by pre-eclampsia in the antepartum; posterior reversible encephalopathy syndrome (PRES)
}

\author{
Yacong Wang ${ }^{1}$, Qinying $\mathrm{CaO}^{2}$, Lihong Zhang ${ }^{3}$, \\ Su'e Zhang ${ }^{2}$, Liang Shi ${ }^{4}$, Ou Sha ${ }^{5}$
}

1. Department of Ophthalmology, The Fourth Hospital of Shijiazhuang, Shijiazhuang 050011, Hebei, China

2. Department of Obstetrics, The Fourth Hospital of Shijiazhuang, Shijiazhuang 050011, Hebei, China

3. Department of Neurology, The Second Hospital of Hebei Medical University, Shijiazhuang 050000, Hebei, China

4. Department of Radiology, The First Hospital of Shijiazhuang, Shijiazhuang 050011, Hebei, China

5. School of Medicine, Shenzhen University Science Health Centre, Guangdong 518060, China

\section{DOI: http://dx.doi.org/10.4314/ahs.v15i2.51}

We present a case report of a patient presenting posterior reversible encephalopathy syndrome (PRES), a rare acute neurological condition associated with pre-eclampsia. A possible common aetiology and successful clinical management approach is reported.

PRES is a rare neurological syndrome first described in $1996^{1}$. Its clinical manifestation varies and is nonspecific ${ }^{1}$. Most PRES patients suffer from headaches, consciousness disorders, seizures, vomiting, mental status changes and visual abnormalities ${ }^{1}$. Its typical imaging finding is characterized by a bilateral posterior cerebral vasogenic edema, especially in the parietal lobe and occipital lobe ${ }^{1,2}$. Pre-eclampsia or eclampsia is the most

\author{
Corresponding authors: \\ Lihong Zhang \\ Department of Neurology \\ The Second Hospital of Hebei \\ Medical University \\ Shijiazhuang 050000 \\ Hebei, China \\ Email: lihong.zhang@yahoo.com \\ Tel: +86-311-66007336 \\ Fax: 311-66007448 \\ Ou Sha \\ Department of Medicine \\ School of Medicine \\ Shenzhen University Health Science Centre \\ Shenzhen 518060 \\ Guangdong, China \\ Email:shaou@szu.edu.cn \\ Tel: +86-755-8667 1918 \\ Fax: +86-755-8667 1906
}

common cause of PRES ${ }^{3}$. Most PRES cases associated with pre-eclampsia/eclampsia are postpartum and intrapartum, rather than antepartum ${ }^{4}$. Visual abnormalities in PRES commonly include blurred vision, hemianopia, visual neglect, etc ${ }^{1}$. Reversible cortical blindness is a very rare symptom ${ }^{5}$.

A 23-year-old woman (gravida 2, para 1) at week $37^{+3}$ of gestation was admitted to the Department of Obstetrics of the Fourth Hospital of Shijiazhuang, China, with bilateral visual loss accompanied by headache, nausea and vomiting with unknown reasons for the past 4 hours. The first and second trimester had been uneventful with no history of hypertension and epilepsy before or during pregnancy. There was no other significant history.

On admission, all vital signs were normal except her blood pressure which was 175/107 mmHg. An obstetric examination confirmed a fetus in a head-down position. A color doppler ultrasound showed a single live fetus with intrauterine growth retardation and oligohydramnios, and a laboratory test showed proteinuria $(3+)$. A diagnosis of severe pre-eclampsia was made. Continuous infusion of magnesium sulphate at a dose of $0.5 \mathrm{~g} / \mathrm{h}$ was immediately administered to the patient to relieve vasospasm, and nifedipine and labetalol were administered orally to control blood pressure.

As a result of the reported bilateral visual loss, fundoscopic examination was performed by an ophthalmologist and slight narrowing of the retinal artery was detected. Cortical blindness was suspected and a cranial MRI examination with diffusion-weighted imaging (DWI) was performed with a $1.5 \mathrm{~T}$ scanner (Achieva, Philips Healthcare, Best, The Netherlands). An 8-chan- 
nelled head-neck coil was used as signal transmitter and images (T2WI) and fluid-attenuated inversion recovery receiver. The image showed low signal intensity in the (FLAIR). Isointensity or slightly increased sionals were bilateral frontal lobes, parietal lobes, temporal lobes and found through DWI. Hyperintense signals showed ap occipital lobes in T1-weighted images (T1WI). In con- parent diffusion coefficient (ADC) maps (Fig. 1), sugtrast, hyperintense signals were found in T2-weighted gesting a diagnosis of PRES.

Fig. 1 Axial MR imaging in PRES patient with pre-eclampsia. A, T1WI shows roughly symmetric low signal intensity located in the bilateral frontal lobes, parietal lobes, temporal lobes and occipital lobes (arrows); B and C, T2WI and FLAIR sequence, respectively, both of which show hyperintense signals in the same positions indicated by arrows; D, DWI shows slightly increased signals in the frontal lobes and isointense signals in the other lobes (arrows); E, ADC maps reveals hyperintensities in similar regions (arrows), which are consistent with the images in vasogenic edema.

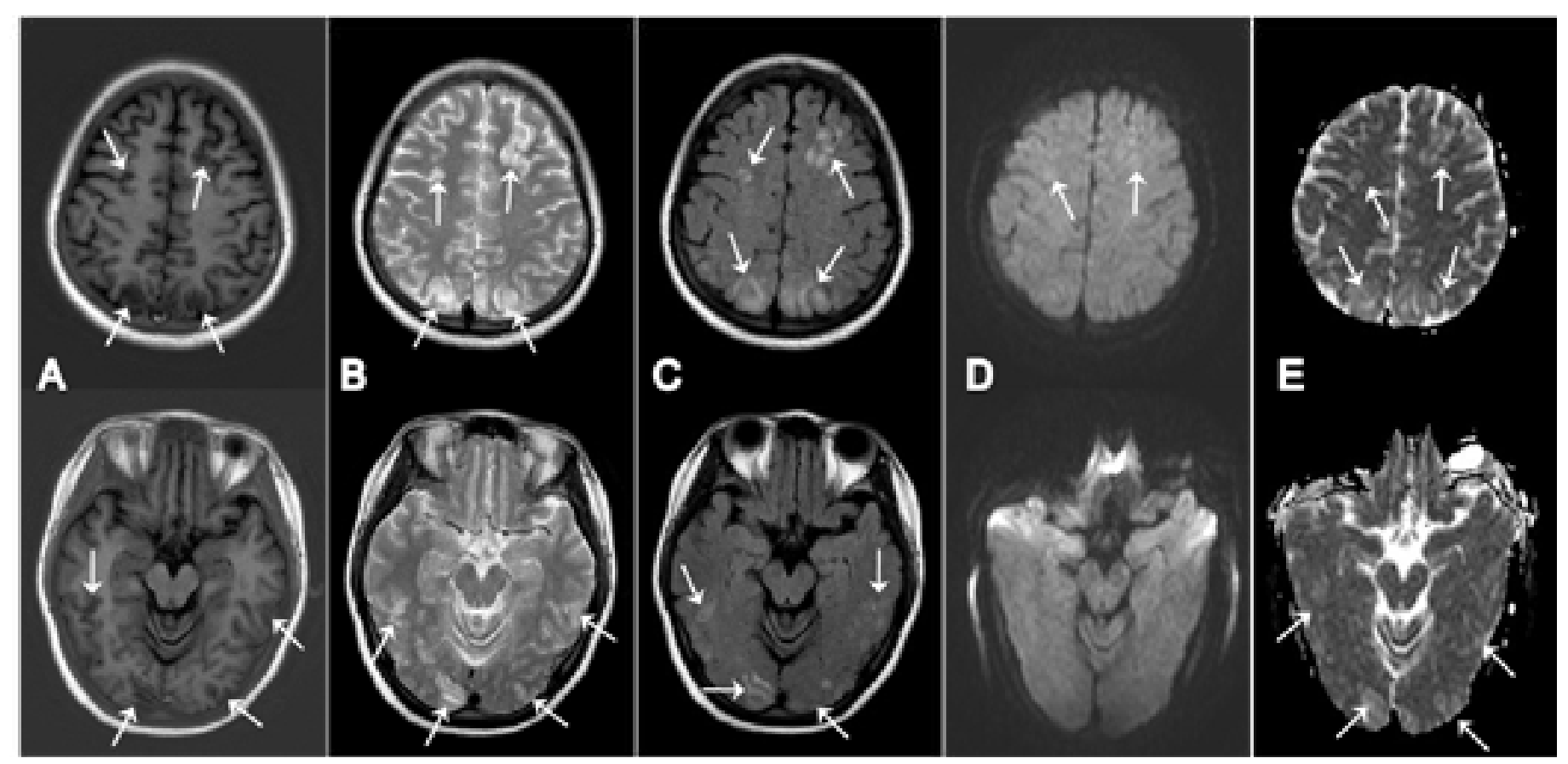

After controlling the blood pressure, the patient under- she was discharged from the hospital. A final diagnosis went a caesarean section 6 hours after admission to the of PRES with severe pre-eclampsia was confirmed. hospital, and delivered a 1450 gram baby. The subsequent pathological examination showed inflammatory The pathophysiological mechanism of PRES caused cell infiltration in the chorion of the placenta. Some by pre-eclampsia is still unclear. One hypothesis is that of the chorion stroma had become marginally fibrotic, high blood pressure causes cerebrovascular autoregulasome showing fibrous necrosis. Inflammatory cell in- tion failure, resulting in hyperperfusion. Another is that three hypoperfusion is caused by vasospasm or vasoconstricdays after surgery, the patient's vision recovered, her tion $^{6}$. Vasogenic edema in brain tissue occurs in both headache disappeared, and blood pressure dropped to hypotheses $\mathrm{s}^{6}$. Consequently, there is endothelial damage, 130/61 mmHg. Eight days later, her wound healed and at least in part, at the blood-brain barrier?
The cause of pre-eclampsia is unclear, but is known to be associated with endothelial damage in the placenta ${ }^{8}$ . In this case, necrosis and phagocyte infiltration were found in the placenta. The diffuse vascular endothelial activation and injury, consequent vasoconstriction and an increase in endothelial permeability, may induce the variable signs of pre-eclampsia, including vasogenic edema of brain tissue ${ }^{8,9}$. If vasogenic edema occurs in the visual cortex of the patient, it may induce cortical blindness.

As PRES improved and resolved after delivery of the baby and the placenta, it is suggested that the culprit may be in the placenta ${ }^{3,10}$. The endothelial damage noted in the placenta may be paralleled in the endothelial damage at the blood-brain barrier with a possible common aetiology $y^{8,9}$.

It is imperative that clinicians, including obstetricians, ophthalmologists and neurologists are familiar with variable features and factors of PRES and its association with blindness. A fundoscopy examination is essential to diagnose a patient with pre-eclampsia who complains of ophthalmic symptoms ${ }^{7}$. If cerebral oedema is suspected, an MRI must also be performed. In PRES, the MRI image shows symmetrical or bilateral vasogenic edema in the parietal and occipital lobe. Vasogenic edema is also commonly found in the frontal lobe, temporal lobe and cerebellar hemisphere ${ }^{1,2}$. In the brain edema area, an MRI image shows hypointense or isointense signals in T1WI and hyperintense signals in T2WI and FLAIR sequence ${ }^{2,11}$. The combination of DWI and ADC maps can separate vasogenic edema from cytotoxic edema, which is critical in the diagnosis of PRES ${ }^{11}$. The hypointensive or isointensive signals in DWI and hyperintensive signals in ADC images indicate the area of vasogenic edema. The signals in DWI sometimes increase slightly due to T2 "shine-through" effect in vasogenic edema. In contrast, the hyperintensive signals in DWI and hypointensive signals in ADC images indicate the area of cytotoxic edema. Therefore, DWI and ADC maps can diagnose PRES and early stage cerebral infarction easily, enabling prompt diagnosis, critical to guide therapy and informed prognosis of the patient with PRES ${ }^{12}$

The key to treat pre-eclampsia and PRES include control of blood pressure, prevention of seizures, and termination of pregnancy ${ }^{13}$. Antihypertensive treatment should be given when the patient's systolic pressure is consistently higher than $160 \mathrm{mmHg}$ or diastolic blood pressure is $105-110 \mathrm{mmHg}$ or above. Common antihypertensive drugs include labetalol, hydralazine and short-acting oral nifedipine. The preferred medicine to prevent seizures is magnesium sulphate to relieve vascular spasm. As soon as blood pressure is stabilised, the pregnancy should be terminated immediately ${ }^{13}$.

\section{Conflicts of interest:}

There is no conflict to disclosure.

\section{Acknowledgement}

Grant support from the National natural science foundation of China (No. 81171154), Collaborative innovation program of Shenzhen (GJHS20120621153317134) and Science \& technology support program of Hebei Province (No. 122777142) is gratefully acknowledged. The authors thank Ms Taraneh Afnan-Holmes of Shenzhen University for her native English revision of this manuscript.

\section{References}

1. Hinchey J, Chaves C, Appignani B, Breen J, Pao L, Wang A, et al. A reversible posterior leukoencephalop athy syndrome. New England Journal of Medicine 1996; 334(8): 494-500

. Hugonnet E, Da ID, Boby H, Claise B, Petitcolin V, Lannareix V, et al. Posterior reversible encephalopathy syndrome (PRES): Features on CT and MR imaging. Diagnostic and Interventional Imaging. 2013; 94: 45-52. 3. Liman TG, Bohner G, Heuschmann PU, Endres M, Siebert E. The clinical and radiological spectrum of posterior reversible encephalopathy syndrome: The retrospective Berlin PRES study. Journal Neurology. 2012; 259(1): 155-164.

4. Hegde HV, Patil PB, Rameshkumar R, Sunita TH, Bhat MT, Desai RM, et al. A rare case of antepartum posterior reversible encephalopathy syndrome. Anaesthesia and Intensive Care. 2011; 39 (3): 499-502.

5. Llovera I, Roit Z, Johnson A, Sherman L. Cortical blindness, a rare complication of pre-eclampsia. The Journal of Emergency Medicine. 2005; 29(3): 295-297.

6. Bartynski WS. Posterior reversible encephalopathy syndrome, part 2: Controversies surrounding pathophysiology of vasogenic edema. AJNR American Journal of Neuroradiology. 2008; 29(6): 1043-1049.

7. Pula JH and Eggenberger E. Posterior reversible encephalopathy syndrome. Current Opinion in Ophthalmology. 2008; 19(6): 479-484

8. Redman CW and Sargent IL. Latest advances in understanding preeclampsia. Science. 2005; 308(5728): 1592-1594. 
9. Noris M, Perico N, Remuzzi G. Mechanisms of disease: Pre-eclampsia. Nature Clinical Practice Nephrology. 2005; 1(2): 98-114.

10. Servillo G, Striano P, Striano S, Tortora F, Boccella $\mathrm{P}$, De RE, et al. Posterior reversible encephalopathy syndrome (PRES) in critically ill obstetric patients. Intensive Care Medicine. 2003; 29(12): 2323-2326.

11. Doelken M, Lanz S, Rennert J, Alibek S, Richter G, Doerfler A. Differentiation of cytotoxic and vasogenic edema in a patient with reversible posterior leukoencephalopathy syndrome using diffusion-weighted MRI. Diagnostic and Interventional Radiology. 2007;13(3): 125-128.

12. Nasr R, Golara M, Berger J. Posterior reversible encephalopathy syndrome in a woman with pre-eclampsia. Journal of Obstetrics Gynaecology. 2010. 30(7): 730-732. 13. Sibai B, Dekker G, Kupferminc M. Pre-eclampsia. Lancent. 2005. 365(9461): 785-799. 